Sybille Krämer · Sibylle Schmidt (Hg.)

Zeugen in der Kunst 

Sybille Krämer · Sibylle Schmidt Hg.

\section{Zeugen in der Kunst}

Wilhelm Fink 
Umschlagabbildung:

Dadang Christanto: Litsus (2004), Performance am 31. Mai 2015 am „4A Centre for

Contemporary Asian Art“ in Sydney, im Rahmen von „MASS GROUP INCIDENT:

48HR Incident“. Foto: Pedro de Almeida. Mit freundlicher Genehmigung des Künstlers, sowie Marita Smith von „Gallerysmith“ in Melbourne und dem „4A Centre for

Contemporary Asian Art“ in Sydney.

Gedruckt mit Unterstützung der Deutschen Forschungsgemeinschaft.

Bibliografische Information der Deutschen Nationalbibliothek

Die Deutsche Nationalbibliothek verzeichnet diese Publikation in der Deutschen

Nationalbibliografie; detaillierte bibliografische Daten sind im Internet über http://dnb.d-nb.de abrufbar.

Alle Rechte, auch die des auszugsweisen Nachdrucks, der fotomechanischen Wiedergabe und der Übersetzung, vorbehalten. Dies betrifft auch die Vervielfältigung und Übertragung einzelner Textabschnitte, Zeichnungen oder Bilder durch alle Verfahren wie Speicherung und Übertragung auf Papier, Transparente, Filme, Bänder, Platten und andere Medien, soweit es nicht $\$ \$ 53$ und 54 UrhG ausdrücklich gestatten.

(C) 2016 Wilhelm Fink, Paderborn

(Wilhelm Fink GmbH \& Co. Verlags-KG, Jühenplatz 1, D-33098 Paderborn)

Internet: www.fink.de

Einbandgestaltung: Evelyn Ziegler, München

Printed in Germany

Herstellung: Ferdinand Schöningh GmbH \& Co. KG, Paderborn

ISBN 978-3-7705-6020-2 
Michael Bachmann

\section{SPIELE MIT ZEUGEN \\ Zur GERICHTSBARKEIT DER BÜHNE IM GEGENWARTSTHEATER}

In seinem Versuch, die „Schaubühne als [...] moralische Anstalt“ zu begründen, behauptet Schiller 1784 eine „Gerichtsbarkeit der Bühne“, die dort beginne, wo „das Gebiet der weltlichen Gerichte sich endigt. “1 Damit wird - sicher auch aus strategischen Gründen - eine Dramentheorie entworfen, die strikt moralischen Imperativen folgt und den Platz legitimen Theaters in der bzw. für die Gesellschaft nach dessen Fähigkeit bestimmt, durch die sinnliche Verkörperung abstrakter und vermeintlich universaler Gesetze die Menschheit zu mehr Gerechtigkeit zu führen; freilich nur um den Preis dessen, was Wolfgang Schäffner und Joseph Vogl für das späte 18. Jahrhundert als parallel statthabende „Verpolizeilichung der Ästhetik“ und „Ästhetisierung der Polizei“ untersucht haben: „Während der grobe Raster von Recht und Gesetz nur eine negative Aktionsweise impliziert, begreift sich das Schillersche Bühnenwesen als Interventionsraum, der [...] , die Gerichtsbarkeit bis in die verborgensten Winkel des Herzens' fortsetzt. " 2

Im Folgenden geht es mir nicht darum, eine historische Genealogie von Schillers „Gerichtsbarkeit“ zu Berufungen auf eine „Gerichtsbarkeit der Bühne“ im Gegenwartstheater seit den 1960er Jahren aufzuzeigen. Im Kontext der Frage nach künstlerischer Zeugenschaft interessiert mich jedoch das von Schiller angesprochene Verhältnis von Bühne und Gericht in Dramen und Theaterprojekten, die mit testimonialen Dramaturgien arbeiten: Welche „Gerichtsbarkeit" wird durch die Überführung von Zeugen auf die Theaterbühne behauptet? Was ist ihre Funktion?

\section{Das Kongo Tribunal (2015) zwischen Theater- und Rechtsgeschichte}

An insgesamt sechs Verhandlungstagen zwischen Mai und Juni 2015 tagte im ostkongolesischen Bukavu und in Berlin ein Tribunal, das sich mitunter zur Aufgabe gesetzt hatte, die Verantwortung multinationaler Unternehmen sowie der internationalen Staatengemeinschaft für die seit den 1990er Jahren anhaltende Gewalt auf dem Gebiet der Demokratischen Republik Kongo zu klären. Leitfragen für die an drei exemplarischen Konflikten verhandelte Untersuchung betrafen etwa die „Roh-

1 Friedrich Schiller: Vom Pathetischen und Erhabenen: Schriften zur Dramentheorie, hg. von Klaus L. Berghahn, Stuttgart 2005, S. 4.

2 Wolfgang Schäffner/Joseph Vogl: „Polizey-Sachen“, in: Walter Hinderer (Hg.): Friedrich Schiller und der Weg in die Moderne, Würzburg 2006, S. 47-66, hier S. 57. Das Zitat im Zitat findet sich bei Schiller: Vom Pathetischen und Erhabenen (Anm. 1), S. 4. 
stoff- und Energiepolitik Europas" und sollten ermitteln, ob die am Mineralienabbau im Kongo beteiligten „Unternehmen mitverantwortlich für die Menschenrechtsverletzungen“ in der Region seien oder ob „sie im Gegenteil zu [ihrer] Stabilisierung " beitragen. ${ }^{3}$ Unter dem Vorsitz des belgischen Juristen Jean-Louis Gilissen hörte die international zusammengestellte Jury des Kongo Tribunals rund sechzig Zeugen und Experten - darunter kongolesische Minenarbeiter, Politiker, Militärs und Juristen -, um in ihrem abschließenden Urteil u.a. die im Kongo stationierten UN-Truppen scharf zu kritisieren. Diese hätten „die Bevölkerung nicht umfassend vor Angriffen geschützt" und seien „so ihrem Auftrag nicht gerecht" geworden; auch müsse die „Rolle der Weltbank und anderer Institutionen“ im Zusammenhang mit mutmaßlichen Wirtschaftsverbrechen in der Region ,juristisch und politisch" weiter untersucht werden. ${ }^{4}$

Weder die hier zitierten noch weitere von den Jurys in Berlin und Bukavu ausgesprochene Beschlüsse, Befunde und Kritikpunkte haben eine direkte rechtliche Konsequenz. Dem Tribunal fehlt jede legale Autorität und Jurisdiktion im konventionellen Sinne, die seine Äußerungen zu ,geglückten 'Sprechakten nach J. L. Austin machen würden. ${ }^{5}$ Ein herkömmliches Urteil - auch wenn es falsch sein sollte und ggf. in höherer Instanz aufgehoben würde - ist in seinem Vollzug zunächst performativ im Sinne Austins: Das Urteil konstituiert Wirklichkeit, indem es z.B. eine Angeklagte freispricht. Dem gegenüber besitzt die Forderung des Kongo Tribunals nach einer weiteren Untersuchung der Rolle der Weltbank, selbst wenn sie eine solche Untersuchung mittel- oder langfristig begünstigen sollte, keine performative Effektivität in sich selbst.

Dennoch ist die Besonderheit des Kongo Tribunals nicht primär darin zu suchen, dass es keine konventionelle Jurisdiktion besitzt. Das gilt für eine Reihe von legalen Institutionen insbesondere im Kontext von nationalen Versöhnungsprozessen, die oft von Regierungsseite eingesetzt werden, um historisches und anhaltendes Unrecht gegenüber bestimmten Bevölkerungsgruppen aufzuarbeiten. So untersucht das Waitangi Tribunal seit 1975 die im Zuge der Kolonisierung Neuseelands begangenen Rechtsbrüche des 1840 zwischen der britischen Krone und verschiedenen Māori-Stämmen geschlossenen Vertrags von Waitangi. Dabei wird die historisch vernachlässigte Māori-Fassung des zweisprachigen Vertrags nicht mehr einfach als problematische Übersetzung des englischen Originals verstanden, sondern die dort verwendeten indigenen Rechtsbegriffe in ihrer Differenz zum common law ernstge-

3 „Pressemappe zum Kongo Tribunal“ (2015), S. 9. Zu finden unter www.the-congo-tribunal. com/wp-content/uploads/2015/05/150430_Kongo-Tribunal_pressemappe-1.pdf (letzter Zugriff: 30.8.2015). Das Tribunal wurde vom 29.-31.5.2015 in Bukavu sowie vom 26.28.6.2015 in Berlin durchgeführt.

4 Colette Braeckman u.a. „Berliner Urteil“ (2015), S. 2f. Zu finden unter www.the-congotribunal.com/wp-content/uploads/2015/20150629_Berliner-Urteil_Das-Kongo-Tribunal_ dt.pdf (letzter Zugriff: 30.8.2015).

5 Vgl. J. L. Austin: How to do things with words, hg. von J. O. Urmson/Marina Sbisà, 2. Aufl., Oxford 1975. 
nommen und aufgewertet. ${ }^{6}$ Die Befunde des Tribunals sind jedoch nicht rechtsbindend. Sie werden lediglich in Form von Empfehlungen an die Regierung weitergeben, die dann z.B. darüber entscheidet, ob und in welcher Höhe Entschädigungen gezahlt werden.

Dem Kongo Tribunal fehlt ein solcher Auftraggeber und offizieller Adressat für seine Empfehlungen; keine Regierungsinstitution hat es ins Leben gerufen. Trotzdem liegt die eigentliche Besonderheit des Tribunals auch nicht in diesem Umstand begründet. Für ihn findet es seine Vorläufer z.B. in den 1966 erstmals eingerichteten Russell Tribunalen, auf die es sich explizit beruft. ${ }^{7}$ Auf Initiative des britischen Philosophen Bertrand Russell und unter der Geschäftsführung Jean-Paul Sartres untersuchte das erste Russell Tribunal von 1966/67 auf insgesamt drei Sitzungen in London, Stockholm und Roskilde - die amerikanische Kriegsführung in Vietnam. ${ }^{8}$ Sartres Eröffnungsrede zufolge bezog es seine vermeintliche Unabhängigkeit und Legitimität gerade aus der Tatsache, dass ihm jede Rechtskraft fehlte und es von keiner Macht außerhalb seiner selbst einberufen wurde.

Um mich der im vorliegenden Band verhandelten Frage nach den Bedingungen und Funktionen künstlerischer Zeugenschaft - und ihrer Spezifika im Theater anzunähern, stelle ich zunächst die Theatralität dieser juridischen Selbstermächtigung im Vergleich zur Operationsweise des Kongo Tribunals dar. Der entscheidende Unterschied zwischen diesem Tribunal und seinen, Vorgängern' liegt nämlich darin, dass es sich bei ihm um eine Theaterperformance (des Schweizer Künstlers Milo Rau) handelt. Unter kritischer Bezugnahme auf Austin fokussiere ich das Verhältnis von Spiel, performativem Akt und Schauspiel, das auch für den engeren Bereich (theatraler) Zeugenschaft grundlegend ist.

\section{Tribunal und Theater: Das Spiel performativer Akte}

Wie David Torell argumentiert, erzeugt die Logik des Russell Tribunals eine Zweigerichtetheit der Kritik, die sich zum einen gegen die Kriegsanstrengungen in Vietnam wendet, zum anderen aber gegen die Institutionen des internationalen Rechts, deren Untätigkeit die „Unberufenen“ und „Machtlosen“ des Tribunals zum Han-

6 Vgl. Richard Benton/Alex Frame/Paul Meredith: Te Mātāpunenga: A Compendium of References to the Concepts and Institutions of Mãori Customary Law, Wellington 2013.

7 Vgl. Milo Rau: „Für nichts gestorben“, in: taz: die tageszeitung, 28.1. 2015, S. 16. Zur Geschichte der Russell und anderer nicht von Regierungen eingerichteter Tribunale sowie einer Einschätzung ihrer Wirksamkeit aus Menschenrechtsperspektive vgl. Arthur W. Blaser: „How to Advance Human Rights without Really Trying: An Analysis of Nongovernmental Tribunals“, in: Human Rights Quarterly 14 (1992) 3, S. 339-370.

8 Vgl. Bertrand Russell/Jean-Paul Sartre: Das Vietnam-Tribunal oder Amerika vor Gericht, Reinbek bei Hamburg 1968 u. Bertrand Russell/Jean-Paul Sartre: Das Vietnam-Tribunal II oder Die Verurteilung Amerikas, Reinbek bei Hamburg1969. 
deln zwinge. ${ }^{9}$ Torells Interpretation betont die theatral-performative Dimension dieses Protestakts, die erstens - darauf komme ich später zurück - in der Fundierung des Urteils auf Zeugenaussagen statt auf Dokumenten beruht. ${ }^{10}$ Zweitens besteht die spezifische Konfiguration von Theatralität und Performativität beim Russell Tribunal im Einnehmen einer gleichsam suspendierten Machtposition: Das Tribunal verkündet ein Urteil, ohne die notwendige Autorität dafür zu besitzen. Mit anderen Worten wird ein Sprechakt vollzogen, der durch seine Form auf Konsequenzen pocht, aber nicht entsprechend institutionalisiert ist.

Mit Elizabeth Burns, Christopher Balme und anderen verstehe ich Theatralität in diesem Zusammenhang zunächst als Wahrnehmungsmodus, der erlaubt, Ereignisse, die per definitionem nicht Theater sind - etwa Gerichtsverfahren - mit Begriffen aus dem Diskursfeld Theater zu beschreiben. ${ }^{11}$ Dies kann wertneutral geschehen, ist aber häufig in komplexe Hierarchien der Auf- und Abwertung eingebunden. Für die westliche Rechtsgeschichte etwa arbeitet Julie Stone Peters eine grundlegende Ambivalenz gegenüber dem Theatralen heraus, die insbesondere die Bereiche Beweisführung, Zeugenschaft und Zuschauerorientierung betrifft. Die von ihr untersuchten Texte fasst sie wie folgt zusammen:

„[C]riminal trials must be public, but not too public; show trials are bad, but secret trials are bad as well; evidence must be relevant, but not so dramatic as to be too relevant (no mutilated bodies on the legal stage); testimony must be live, but not be too lively (witnesses must stay in the box); what witnesses say and show should move juries, but it shouldn't move them too much. ${ }^{\text {"12 }}$

Austin folgend rückt das Russell Tribunal durch seinen zugleich spielerischen und ernsthaften Sprechakt - das Urteil aus suspendierter Machtposition - in ,gefährliche' Nähe zum Theater. Bekanntermaßen gilt dem britischen Sprachphilosophen die Bühne als paradigmatischer Ort, der performative Äußerungen zum Scheitern bringe. Diese seien „in a peculiar way hollow or void if said by an actor on the stage, or if introduced in a poem, or spoken in soliloquy. "13 Austins Opposition zwischen ,normalem ' und ,parasitärem 'ästhetischem Sprachgebrauch ist jedoch u.a. deshalb nicht aufrecht zu erhalten ${ }^{14}$, da der institutionelle Rahmen, auf dem die performative Effektivität des Sprechakts beruht, seine eigene Rechtmäßigkeit inszenatorisch

9 Vgl. David Torell: „Remember the Russell Tribunal?“, in: Anna Reading/Tamar Katriel (Hg.): Cultural Memories of Nonviolent Struggles: Powerful Times, Basingstoke 2015, S. 111127, hier S. $114 \mathrm{f}$.

10 Vgl. Ebd., S. 114.

11 Vgl. Elizabeth Burns: Theatricality: A Study of Convention in the Theatre and in Social Life, Harlow: 1972 u. Christopher Balme: Pacific Performances: Theatricality and Cross-Cultural Encounter in the South Seas, Basingstoke: 2007, S. 2-6.

12 Julie Stone Peters: „Legal Performance Good and Bad“, in: Law, Culture and the Humanities 4 (2008), S. 179-200, hier S. 199.

13 Austin: How to do things with words (Anm. 5), S. 22.

14 Vgl. insb. Jacques Derrida: Marges de la philosophie, Paris1972 u. Andrew Parker/Eve Kosofsky Sedgwick: „Introduction: Performativity and Performance“, in: Andrew Parker/Eve Kosofsky 
behaupten muss. Gesetzliche Autorität, so Thomas Weitin, braucht eine „symbolische Form der Repräsentation" - je nach Rechtssystem z.B. eine bestimmte Kostümierung von Anwalt und Richter, das Sich-Erheben vor dem Gesetz, etc. - um den Rahmen zu erzeugen, ,innerhalb dessen performative Rechtsakte in unmissverständlicher Weise vollzogen werden können "; ${ }^{15}$ ein Umstand, der dadurch noch verkompliziert wird, dass die Gesetzeskraft, die dem Urteilsspruch vorausliegt und auf deren Autorität er sich beruft, immer erst durch diesen aktualisiert und von neuem behauptet wird. ${ }^{16}$ Ein weiterer Faktor, der legale Prozesse und Theater einander annähert, besteht im Bezug auf eine zuschauende Öffentlichkeit, sofern die Verfahren - wie in modernen Rechtssystemen üblicherweise der Fall - ein Ideal der Transparenz verfolgen: ,justice must not only be done but must be seen to be done. ${ }^{\text {"17 }}$

Die Jurymitglieder des Russell- und des Kongo Tribunals spielen ihre Rollen in einem anderen Sinne als dies bei Richtern oder Geschworenen in konventionellen Rechtsverfahren der Fall ist, wo es sich - nach Erving Goffman - um kontextabhängiges Alltagstheater bzw. soziologisches Rollenspiel (the presentation of self in everyday life) handelt. ${ }^{18}$ Dennoch sind sie ebenso wenig Schauspieler im Sinne Austins, der auf ein dramatisches Rollenverständnis abzielt, bei dem der Sprechakt scheitert, weil er ein Zitat ist: Die von Austin vorgestellten Darsteller improvisieren nicht; sie sprechen Zeilen, die ihnen vor-geschrieben werden. Die Beteiligten des Russell- und Kongo Tribunals spielen, da sie Rollen einnehmen, für die ihnen die institutionelle Legitimation fehlt, aber ihre Sprechakte behaupten eine andere Ernsthaftigkeit als die von Schauspielern im dramatischen Theater. Darauf beruht die oben beschriebene Zweigerichtetheit der Kritik: Ein echtes Urteil wird getroffen, das sich ernsthaft auf die Situation in Vietnam bzw. im Kongo bezieht, doch weil das Urteil als Spiel suspendiert ist, übt es institutionelle Kritik an jenen, die kein staatlich oder transnational legitimiertes Tribunal einberufen.

Obwohl Russell- und Kongo Tribunal in diesem Sinne parallel operieren, kommt hier der eigentliche Unterschied zwischen dem einen und den anderen Verfahren ins Spiel. Egal wie sehr sie sich Theater wegen der inhärenten Theatralität legaler Prozesse und - beim Russell Tribunal - durch fehlende Legitimation annähern, handelt es sich bei keinem der genannten Beispiele tatsächlich um Theater. Die metaphorische Distanz zwischen Bereichen, die es erlaubt, legale Verfahren als

Sedgwick (Hg.): Performativity and performance, New York ; London: Routledge 1995, S. $1-18$.

15 Thomas Weitin: „Dichter und Richter: Probleme des Urteilens im 18. Jahrhundert“, in: Rechtsgeschichte: Zeitschrift des Max-Plank-Instituts für Europäische Rechtsgeschichte 6 (2005), S. 143-160, hier S. 150.

16 Vgl. ebd., S. 151. Weitin bezieht sich hier auf Jacques Derrida: „Force de loi: Le ,fondement mystique de l'autorité / Force of Law: The ,Mystical Foundation of Authority “", in: Cardozo Law Review 11 (1990), S. 920-1045.

17 Hannah Arendt: Eichmann in Jerusalem: A Report on the Banality of Evil, rev. u. erw. Aufl., New York 1994, S. 277; meine Hervorhebung. Arendt zitiert hier einen bekannten Aphorismus, der sich z.B. im Urteil $R v$ Sussex Justices, exp McCarthy (1923) findet.

18 Vgl. Erving Goffman: The Presentation of Self in Everyday Life, Garden City, NY 1959. 
„theaters of justice" und „spectacles of legality" zu beschreiben, ohne ihnen dadurch zugleich die vermeintliche Konsequenzlosigkeit ästhetischer Rahmung vorzuwerfen, bleibt erhalten. ${ }^{19}$ Das Kongo Tribunal ist anders. Es ist eine Theaterperformance, ein Kunstprojekt des 1977 geborenen Schweizer Regisseurs Milo Rau und seines International Institute of Political Murder. ${ }^{20}$ Nichtsdestotrotz werden - wie beim Russell Tribunal - echte Zeugen gehört, ist die Jury mit Experten besetzt und das Resultat eine Empfehlung bzw. Verurteilung ohne juristische Legitimation.

Was also verändert die theatrale Rahmung des Kongo Tribunals? Was bedeutet sie für die Frage künstlerischer Zeugenschaft? Ausgehend von Milo Raus Theaterprojekt untersuche ich im Folgenden das Spannungsverhältnis zwischen den - wie gerade das Kongo Tribunal zeigt - nicht durchweg klar differenzierten Bereichen der Zeugenschaft in künstlerischen und außer-künstlerischen (z.B. legalen) Kontexten. Dabei skizziere ich, mit Blick jeweils auf die Konstellation zwischen einem legalen Verfahren und einer Theaterproduktion, drei unterschiedliche Funktionalisierungen von künstlerischer Zeugenschaft: Peter Weiss' Die Ermittlung (1965) lese ich mit Bezug auf den Eichmann-Prozess in Jerusalem (1961) als Versuch, auf dem Theater eine Form der Zeugenschaft zu erzeugen, die für sich in Anspruch nimmt, besser der Ermittlung von Wahrheit dienlich zu sein als die Zeugenschaft überlebender Opferzeugen vor Gericht. Anna Deavere Smiths Twilight: Los Angeles, 1992 (1994) hingegen, das die Unruhen nach dem Rodney King-Prozess untersucht, konfiguriert das Verhältnis von Schauspiel und Zeugenschaft - so meine These zugunsten einer Erfahrbarmachung von Differenz. Ubu and the Truth Commission (1997) der Handspring Puppet Company (Text: Jane Taylor; Regie: William Kentridge) verwendet Zeugenschaft auf dem Theater - durch den Einsatz von Puppen zu Gehör gebracht -, um gegenüber der südafrikanischen Truth and Reconcilation Commission (1996-2002) einen Metakommentar zur (Un-)Möglichkeit von Gerechtigkeit geltend zu machen.

19 In ihrer Studie zum Verhältnis von traumatischen Ereignissen und Gerichtsbarkeit greift Shoshana Felman häufig auf die geläufige Metapher des „theaters of justice“ zurück (Shoshana Felman: The Juridical Unconscious: Trials and Traumas in the Twentieth Century, Cambridge, Mass. 2002); die Formulierung „spectacle of legality“ findet sich bei Lawrence Douglas, der damit die Nürnberger Prozesse beschreibt: Neben der Bestrafung von nationalsozialistischen Verbrechen wäre es bei diesen auch darum gegangen, die Neutralität des Gesetzes ,aufzuführen" (Lawrence Douglas: The Memory of Judgment: Making Law and History in the Trials of the Holocaust, New Haven/London 2001). Zur Frage der metaphorischen Distanz im Vergleich von Theater und Gericht vgl. Cornelia Vismann: „,Rejouer les crimes': ,Theater vs. Video“", in: Cardozo Studies in Law and Literature 11 (1999) 2, S. 119-135, hier S. 169.

20 Zum Kongo Tribunal und anderen Projekten des Regisseurs vgl. die Webseite des International Institute of Political Murder (http://international-institute.de/). Rau hat sich in einigen seiner Arbeiten mit dem Verhältnis von Theater, Performance und Rechtsverfahren beschäftigt. Insbesondere sind hier die Moskauer und Zürcher Prozesse (2012-13), Die letzten Tage der Ceausescus (2010-11) sowie Breiviks Erklärung (seit 2012) zu nennen. 


\section{Schauspiel und Zeugenschaft}

Schon ein flüchtiger Blick auf das Kongo Tribunal lässt ein Spezifikum von Theater im Zusammenhang mit künstlerischer Zeugenschaft erkennen: Anders als Film, Literatur, Hörspiel und andere Kunstformen erlaubt es einen Auftritt von Zeugen in analoger Weise zu deren Auftritt vor Gericht, ohne durch Schauspieler, Stimmen, bewegte Bilder oder Texte „vertreten“ zu werden, in leiblicher Ko-Präsenz vor einem gleichzeitig anwesenden Publikum. ${ }^{21}$ So wie etwa das bekannte Performancekollektiv Rimini Protokoll in vielen seiner Arbeiten „Experten des Alltags“ auf die Bühne bittet, die sich selbst spielen, ${ }^{22}$ lässt Raus Kongo Tribunal die Zeugen in ihren eigenen Rollen auftreten. Kommt es bei Rimini Protokoll jedoch zu einem bewusst eingesetzten Spannungsverhältnis zwischen theatraler Wiederholung und außertheatraler Referenz - die ,Experten' folgen einem Skript, das zunächst auf Grundlage ihres Lebens erstellt wurde -, sind die Zeugen bei Rau so frei bzw. gesteuert wie vor Gericht: Statt einen vorher geschriebenen Text zu spielen, sind sie in die dialogische Struktur der Anhörung eingebettet. Der Inszenierungsaspekt, der bei Rimini Protokoll durch die Spielweise der „Experten“ offengelegt wird, wird im Kongo Tribunal durch die szenischen Rahmungen der Zeugenaussagen verdeutlicht. Hier ist etwa die Anwesenheit des Regisseurs mit Kamerateam zu nennen, der - in Vorbereitung auf die zu erstellende Filmfassung - vor jeder Sitzung des Tribunals „Action“ ruft, sowie die Anwesenheit der österreichischen Dramatikerin Kathrin Röggla als „Gerichtsschreiberin“ bei den Berliner Hearings. In einem Live-Blog, der zugleich auf eine Leinwand über der Bühne projiziert wird, liefert sie mehr als nur eine Zusammenfassung der Aussagen und Diskussionen.

Als Metakommentare beharren Rögglas Texte auf dem theatralen Rahmen der Performance und damit auf der ästhetischen Differenz zum Russell Tribunal bei aller Ähnlichkeit der Prozeduren. Am Nachmittag des 27. Juni 2015 weist Röggla „in [ihrer] Funktion als Gerichtsschreiberin“ darauf hin, „dass wir hier in einem Theatersaal sitzen und nicht in einem Gerichtssaal. Sie müssen den Anweisungen des Tribunalleiters nicht Folge leisten. Stehen Sie also nicht auf, wenn Sie dazu aufgefordert werden! “23 Als die Zuschauer sich wenig später doch wie vor Gericht erheben, interpretiert die Dramatikerin dies als „Sog des Naturalismus“, dem auch sie erliege; er drücke „einen Wunsch nach Ermächtigung aus. Der Wunsch nach der Produktion von Gerechtigkeit. Dieses Tribunal ist eine Show, aber sie verlangt nach Konsequenzen. " 24

21 Vgl. Erika Fischer-Lichte: Ästhetik des Performativen, Frankfurt a.M. 2004, S. 58-62 zur leiblichen Ko-Präsenz als Grundbestimmung von Theater.

22 Vgl. z.B. Miriam Dreysse/Florian Malzacher (Hg.): Experten des Alltags: Das Theater von Rimini Protokoll, Berlin 2007 u. Johannes Birgfeld/Ulrike Garde/Meg Mumford (Hg.): Rimini Protokoll Close Up: Lektüren, Hannover 2015.

23 Kathrin Röggla, „Live Ticker [zum Kongo Tribunal]“ (2015), o. S. Zu finden unter www.thecongo-tribunal.com/?page_id=267 (letzter Zugriff: 30.8.2015).

24 Ebd., o. S. 
In kritischer Auseinandersetzung mit dem Dokumentartheater der 1960er Jahre schrieb Peter Handke 1967 über den Einbezug nicht-professioneller Schauspieler auf der Bühne, dass ,wenn, wie kürzlich in Oberhausen, im Theater ,echte' Kumpels auftreten und dort ein Protestlied anstimmen“ dies "falsch und unwahr" werde. ${ }^{25}$ Der Grund hierfür liege in einer Formalisierung des Realen durch den Bedeutungsraum Theater. Dieser sei so „bestimmt, dass alles, was außerhalb des Theaters

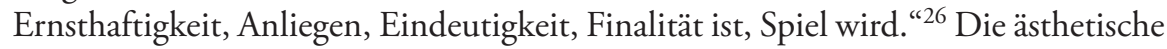
Formalisierung der Zeugenschaft im Kongo Tribunal, die wohlgemerkt weder deren Inhalt noch deren Funktionsweise betrifft, operiert auch hier analog zum Russell Tribunal: Dass ein Prozess ernsthaft, aber im Spiel bzw. ohne Legitimation außerhalb seiner selbst vollzogen wird, fördert gerade erst den "Wunsch nach der Produktion von Gerechtigkeit“" (Röggla) bzw. gibt ihm einen Ort. So kam es in Bukavu nach der Performance zu Protesten: „,Wir wollen ein richtiges Tribunal!““27

Beruht die politische Effektivität von Raus Theater-Tribunal einerseits tatsächlich auf der von Handke beschriebenen Formalisierung des Realen - d.h. der Verschiebung des Zeugnisses wie des gesamten legalen Verfahrens in den ästhetischen Rahmen -, ist sie andererseits davon abhängig, dass Theater - anders als Handke mit Blick auf eine historisch spezifische theatrale Praxis postuliert - kein ,reines Spiel ist. Theater und Performancekunst werden daher oft als privilegierte Orte künstlerischer Zeugenschaft konzeptualisiert - etwa bei Freddie Rokem und HansThies Lehmann -, weil in ihnen die Grenzen zwischen fiktionaler Darstellung und realer Handlung besonders prekär sind. „Im Unterschied zu allen Künsten des Objekts und der medialen Vermittlung", schreibt z.B. Lehmann, finden im Theater „sowohl der ästhetische Akt selbst (das Spiel), als auch der Akt der Rezeption (der Theaterbesuch) als reales Tun in einem Hier und Jetzt statt. ${ }^{\text {"28 }}$ Rokem zufolge können Schauspieler zu effektiven Bindegliedern zwischen einer abwesenden Wirklichkeit und dem „Hier und Jetzt“ der Theateraufführung werden, da Theater - anders als Film, der die Schauspieler vor der Projektion in „Bilder“ verwandelt - in leiblicher Ko-Präsenz vor sich geht. Da Schauspieler zugleich (eine Rolle) spielen und (selbst) handeln, können an ihnen die Grenzen zwischen ontologischen Sphären - dem Ästhetischen und dem Sozialen, Geschichte und Gegenwart, etc. - zusammenbrechen..$^{29}$ Dabei stellt sich die Frage, welche ethischen Implikationen es hat, wenn Zeugen auf die Bühne überführt werden - wie beim Kongo Tri-

25 Peter Handke: „Straßentheater und Theatertheater“, in: Peter Handke (Hg.): Prosa Gedichte Theaterstücke Hörspiel Aufsätze, Frankfurt a.M.: Suhrkamp 1969, S. 303-307, hier S. 304.

26 Ebd., S. $304 f$.

27 Andreas Tobler: „Das Kongo Tribunal“, in: Die Zeit, 1.7. 2015.

28 Hans-Thies Lehmann: Postdramatisches Theater, Frankfurt a.M. 1999, S. 12. Lehmann bezieht seine Ausführungen zum „postdramatischen Theater" auf künstlerische Zeugenschaft in Hans-Thies Lehmann: „Prädramatische und postdramatische Theater-Stimmen: Zur Erfahrung der Stimme in der Live-Performance“, in: Doris Kolesch/Jenny Schrödl (Hg.): KunstStimmen, Berlin 2004, S. 40-66.

29 Vgl. Freddie Rokem: Performing History: Theatrical Representations of the Past in Contemporary Theatre, Iowa City 2000, S. 191. 
bunal - oder Schauspieler den Platz von Zeugen einnehmen, wie sehr viel häufiger der Fall.

Eine Besonderheit des Kongo Tribunals besteht darin, dass es seine Zeugen zwar in den ästhetischen Rahmen überführt, diese aber - anders als die „Experten“ von Rimini Protokoll - nicht zu Schauspielern in dem Sinne macht, dass sie den "Text“ ihrer Erfahrungen wiederholen. Als einmalige Performance, die sich über insgesamt sechs „Verhandlungstage“ erstreckt, ist das Kongo Tribunal auf eine andere Art der Wiederholbarkeit angelegt - zu Raus Projekt gehört ein Dokumentarfilm, der 2017 ins Kino kommen soll - als eine Theaterproduktion mit mehrfachen Aufführungen. Auch deshalb stellt sich das Spannungsverhältnis zwischen legalem Verfahren und theatraler Rahmung, Schauspiel und Zeugenschaft hier anders dar als in den im folgenden skizzierten Beispielen.

\section{Die Ermittlung (1965) - Wahrheitssuche und Erklärbarkeit}

Peter Weiss' Die Ermittlung inszeniert eine abstrahierte Gerichtsverhandlung mit neun namenlosen Zeugen. Diese Art der Umsetzung ist weniger dem Material geschuldet, das der Dramatiker bearbeitet - dem ersten Frankfurter AuschwitzProzess (1963-65) - als bewussten Überlegungen zur Darstellbarkeit der Shoah, die sich auch auf die Frage künstlerischer Zeugenschaft beziehen lassen.

Laut einer Vorbemerkung zur Ermittlung sollen Aufführungen nicht versuchen, den Ort des Frankfurter Auschwitzprozesses nachzubilden: „Eine solche Rekonstruktion erscheint dem Schreiber des Dramas ebenso unmöglich, wie es die Darstellung des Lagers auf der Bühne wäre. “30 Die hier behauptete Unmöglichkeit einer szenischen Darstellung von Auschwitz begründet zunächst den Fokus der Ermittlung auf die sprachliche Ebene: Das Stück funktioniert als Montage von Zeugenaussagen. Zugleich ist hier das Verhältnis von Schauspieler und (Gerichts-)Zeuge angesprochen und damit eine bestimmte Funktionalisierung von künstlerischer Zeugenschaft. Sie basiert auf Weiss' Hoffnung, dass "die Wirklichkeit, so undurchschaubar sie sich auch macht" durch künstlerische Praxis ,in jeder Einzelheit erklärt werden kann“. ${ }^{31}$ Theater könne „Fakten zur Begutachtung "32 vorlegen, wenn es keine Illusion „augenblickliche[r] Wirklichkeit“ biete, sondern „das Abbild von einem Stück Wirklichkeit, herausgerissen aus der lebendigen Kontinuität“ . ${ }^{33}$

In gewisser Weise gilt dies auch für Gerichtsverfahren, welche die Wirklichkeit, auf die sie sich beziehen, nicht "aufführen“, sondern in einer Art szenischem Spiel zwischen Richtern, Anwälten, Angeklagten und Zeugen - verhandeln. Trotzdem besteht Weiss auf der Differenz zwischen seinem Modell dokumentarischen Thea-

30 Peter Weiss: Die Ermittlung: Oratorium in 11 Gesängen, Frankfurt a.M. 1991, S. 9.

31 Peter Weiss: „Notizen zum dokumentarischen Theater“, in ders. (Hg.): Rapporte 2, Frankfurt a.M. 1971, S. 91-104, hier S. 104.

32 Ebd., S. 97.

33 Ebd., S. 95. 
ters und legalen Verfahren, da ersteres keinen Anspruch darauf habe, „der Authentizität eines Gerichtshofs von Nürnberg, eines Auschwitzprozesses in Frankfurt, eines Verhörs im amerikanischen Senat, einer Sitzung des Russell-Tribunals nahezukommen“. ${ }^{34}$ Weiss deutet diesen „Authentizitätsverlust“ positiv, da er eine weitere Entfernung aus der „lebendigen Kontinuität“ der vermeintlich undurchschaubaren Wirklichkeit darstellt. Neben dem Bühnenbild, wie oben skizziert, betrifft das vor allem den Schauspielstil: Die Schauspieler sollen sich nicht einfühlen, sondern lediglich „referieren“, was die Zeugen vor Gericht „ausdrückten“.35 Nach Meinung des Dramatikers führt dies von den „emotionalen Kräften“ vor Gericht zu „Fakten“ auf der Bühne, zu einem „Konzentrat der Aussage“, das keine „persönlichen Erlebnisse und Konfrontationen“ mehr enthalte. ${ }^{36}$

Die Worte „referieren“ und „ausdrücken“ sind in diesem Kontext keineswegs synonym zu verstehen, wie ein Seitenblick auf den Eichmann-Prozess verdeutlicht. ${ }^{37}$ Einer der bekanntesten Augenblicke jenes Verfahrens war die Aussage des Auschwitz-Überlebenden und Schriftstellers Yehiel Dinur alias K-Zetnik, der nach wenigen Minuten im Zeugenstand bewusstlos zusammenbrach. Die Szene, die bis heute im israelischen Fernsehen wiederholt wird, hat für nicht wenige Kommentatoren höchste Symbolkraft erreicht: Dinurs unterbrochenes Zeugnis werde zu einer stummen, aber wirkmächtigen Botschaft, schreibt z.B. Shoshana Felman:

"[T] he witness testifies through his unconscious body. Suddenly, the testimony is invaded by the body. The speaking body has become a dying body. The dying body testifies dramatically and wordlessly beyond the cognitive and the discursive limits of the witness's speech. ${ }^{\text {" }} 8$

Felmans Interpretation löst Zeugenschaft aus dem Versuch einer diskursiven Darstellung dessen, was sich der Darstellung - als Trauma - entzieht. Stattdessen wird Dinurs Aussage als körperliches Einbrechen der Vergangenheit in die Gegenwart begriffen. Hier scheint kein Umweg über die Sprache nötig, um den zu bezeugenden Augenblick mit Hilfe einer Technik zu wiederholen. Felman beschreibt Dinurs Ohnmacht als körperliche Anwesenheit des Abwesenden, die den Bezug zwischen Zuhörern, Zeuge und Ereignis auf einer körperlich-performativen Ebene herstellt.

Das entspricht einem Funktionswandel der Zeugenschaft „nach Auschwitz“, den die Historikerin Anette Wieviorka beschreibt: von einem im engeren Sinn juristischen Zeugnis, das primär ,informieren“ soll, zu einem Zeugnis, bei dem die affektive Übertragung von Erfahrung im Vordergrund steht. ${ }^{39}$ Der von Weiss verwende-

34 Ebd., S. 100.

35 Ebd.

36 Ebd.

37 Vgl. zum folgenden Michael Bachmann: „Theatre and the Drama of Law: A ,Theatrical History' of the Eichmann Trial", in: Law Text Culture 14 (2010), S. 94-116.

38 Felman: The Juridical Unconscious (Anm. 19), S. 163.

39 Vgl. Annette Wieviorka: „The Witness in History“, in: Poetics Today 27 (2006) 2, S. 385397. 
te Begriff des „Referierens“ zielt vor diesem Hintergrund auf ein testimoniales Konzept im juristischen Sinne, das Information und ihre anschließende Beurteilung dominant setzt, während mit „ausdrücken“ ein Verständnis von Zeugenschaft bezeichnet wäre, das die körperlich-performative Dimension betont. Wenn Weiss von den Schauspielern der Ermittlung verlangt, als Zeugen nur zu „referieren“, lässt sich dies mitunter als Versuch verstehen, jenes Paradox zu lösen, dass statt der Zeugen ,nur' Schauspieler auf der Bühne stehen.

Darüber hinaus soll das distanzierte Spiel („referieren“ statt „ausdrücken“) jedoch eine Aussage produzieren, bei der der Zeuge - über den Umweg des Schauspielers zu einem Überbringer von Fakten reduziert wird, die zur Begutachtung vorgelegt werden. Ein solches Modell im engeren Sinne juristischer Zeugenschaft sei auf der Bühne möglich, nicht aber im Gerichtssaal selbst, wo das legale Narrativ in Anwesenheit der überlebenden Opfer-Zeugen und ihrer Traumatisierung zusammenbricht. Weiss spricht in diesem Zusammenhang davon, die Zeugen durch künstlerische Bearbeitung „zu bloßen Sprachrohren“ für Fakten zu machen. ${ }^{40}$ Dieser Logik zufolge erlaubt ausgerechnet Theater als „Ort der schweren Körper“41 eine „Entkörperlichung“ der Aussagen zugunsten der Präsentation von „Wahrheit“. ${ }^{42}$

\section{Twilight: Los Angeles, 1992 (1994) - Erfahrbarmachung von Differenz}

Am 3. März 1991 verprügelten vier Polizisten - drei von ihnen Weiße - den AfroAmerikaner Rodney King bei einer Verhaftung wegen erhöhter Geschwindigkeit. Da sie dabei von einem Anwohner gefilmt wurden, ließ sich beweisen, dass die Beamten King mindestens fünfzig Mal mit Stöcken schlugen und mehrfach traten. Nichtsdestotrotz wurden sie rund ein Jahr später, am 29. April 1992, von einem überwiegend weißen Geschworenengericht freigesprochen. Daraufhin kam es in Los Angeles zu sechs Tage anhaltenden Unruhen, in deren Verlauf 53 Menschen getötet und fast 3000 verletzt wurden.

In ihrem Dokumentartheaterstück Twilight: Los Angeles, 1992 (1994) untersucht die Schauspielerin und Dramatikerin Anna Deveare Smith die Unruhen basierend auf Interviews, die sie mit einer großen Anzahl Beteiligter und Zeitzeu-

40 Weiss: Die Ermittlung (Anm. 30), S. 9.

41 Lehmann: Postdramatisches Theater (Anm. 28), S. 12.

42 Zur Kritik an dieser Vorgehensweise vgl. Vivian M. Patraka: Spectacular Suffering: Theatre, Fascism, and the Holocaust, Bloomington 1999, S. 102: „By disembodying these voices, Weiss [...] annihilates the survivors of genocide. Moreover, the embodying of the voices of the accused (who are not speaking tubes but ,themselves') suggests that the narrative underlying this seemingly unmediated presentation of ,the facts' is actually a narrative of Germany's past and present guilt for the events of fascism and the Holocaust, a narrative paradoxically staged at the expense of survivors and their experience of atrocity." 
gen der unterschiedlichen Konflitkparteien geführt hat. Dabei fallen auf den ersten Blick drei Unterschiede zu Weiss' Konzeption ins Auge: Erstens beruht die Praxis der Entkörperlichung bei der Ermittlung darauf, dass Opferzeugen und Täter klar getrennt sind und die Aussagen der ersteren sich auf eine klare Geschichte - im Sinne der Erklärbarkeit einer nur vermeintlich ,undurchschaubaren ' Welt - reduzieren lassen, während die Aussagen der letzteren nichts zählen: Sie stellen lediglich Ausflüchte dar. Smiths Montage hingegen folgt einem Prinzip der Brüchigkeit und der Singularität. Anders als bei Weiss sind die Zeugen nicht namenlos, sondern besitzen individualisierte Standpunkte, die einander widersprechen, ohne dass diese Konflikte aufgelöst würden. ${ }^{43}$

Dies hat zweitens damit zu tun, dass sich Smith stärker als Weiss vom legalen Modell der Zeugenschaft löst. Zwar ist Twilight: Los Angeles, 1992 auf einen Gerichtsprozess als Auslöser der in Zeugenaussagen nacherzählten Unruhen bezogen und behauptet Theater als Ort der Verhandlung jener Unruhen sowie der größeren gesellschaftlichen Spannungen, die sie verkörpern. Smiths Vorgehensweise entspricht aber weniger einem (abstrahierten) Gerichtsverfahren als dem Modell des Dokumentarfilms mit Zeitzeugen, der von Szene zu Szene schneidet und Interviews in unterschiedlichen Kontexten präsentiert.

Diese Kontexte werden bei Smith nicht im Bühnenbild angedeutet, sondern durch spärliche Requisiten und Kostümwechsel sowie im Spielgestus der Performerin. Der dritte - für das Verhältnis von Schauspiel und Zeugenschaft entscheidende - Unterschied zu Weiss besteht nämlich darin, dass Twilight: Los Angeles, 1992 ein Solostück ist. Alle Zeugen und ihre disparaten Aussagen werden von Smith in schneller Abfolge hintereinander gespielt, wobei sie auch zwischen Zeugen hin- und herschaltet. Wo Weiss einen Einsatz von Schauspielern imaginiert, der das Zeugnis auf seinen Informationsgehalt hin ,reinigt', wählt Smith die von ihr gespielten Aussagen umgekehrt nach ihrer „Körperlichkeit“ aus. Es gehe ihr darum, so die Schauspielerin und Dramatikerin, ,how an interview text works as a physical, audible, performable vehicle. [...] Every person [...] who I perform has a presence that is much more important than the information they give." 44

Smith erklärt ihren Schauspielstil im Unterschied zum psychologischen Realismus, der im Sinne Stanislavskijs versucht, die Umstände einer Figur zu ergründen, nach der Verbindung zu den eigenen Umständen zu suchen und dann zu überlegen, wie man bei der Darstellung der Figur auf eigene Erfahrungen zurückgreifen kann. Smith verweigert sich einem solchen Verstehen und zielt stattdessen auf eine ,Wiederholung' der Figur bzw. des interviewten Zeugen ab. Um dies zu erzielen, arbeitet die Schauspielerin und Dramatikerin nicht mit schriftlichen Protokollen sondern mit Tonaufnahmen, die sie während der Probephase per Kopfhörer mitspricht: „The point is simply to repeat [the testimony] until I begin to feel it and

43 Vgl. Anna Deavere Smith: Twilight: Los Angeles, 1992, New York 1994. 44 Ebd., S. xxiii f. 
what I begin to feel is [the witness's] song and that helps me to remember more about his body. “ 45

An die Stelle von Einfühlung oder Analyse tritt eine Wiederholung von Zeugenschaft, die eine Differenz zu ihren Trägern erlaubt und erfahrbar macht, nicht zuletzt weil Smith auf andere Weise zum ,Sprachrohr ${ }^{`}$ wird als die Schauspieler der Ermittlung. Die Position und Autorität einzelner Zeugenaussagen wird dadurch unterminiert, dass die verschiedenen Standpunkte - und mit ihnen unterschiedliche Stimmlagen, Körpergesten sowie vermeintlich sinnlose Sprachticks (Gähnen, Stottern, Wiederholungen, etc.) - durch eine einzige Performerin ,laufen', deren gleichzeitige Nähe und Differenz zu allen Zeugen eine Begegnung erlaubt, die jenseits von Verstehen liegt.

\section{Ubu and the Truth Commission (1997) - Wahrheit und Gerechtigkeit?}

Ein drittes Modell des Verhältnisses von Zeuge und Schauspieler schlägt die Inszenierung Ubu and the Truth Commission (1997) vor, indem sie mit Puppen, sichtbaren Puppenspielern und Schauspielern arbeitet. Gegenstand der Produktion sind die Erfahrungen der Apartheid und eine kritische Auseinandersetzung mit der Truth and Reconciliation Commission als legaler Institution zur Aufarbeitung jener Zeit.

Bei Ubu and the Truth Commission werden Täterzeugen auf der Bühne von Menschen gespielt, während das Zeugnis der Opfer durch bewusst grob geschnitzte Holzpuppen gesprochen wird. Dem Regisseur William Kentridge zufolge ist dies mitunter die Antwort auf eine ethische Frage:

„[W] hat is our responsibility to the people whose stories we are using as raw fodder for the play? There seemed to be an awkwardness in getting an actor to play the witnesses - the audience being caught halfway between having to believe in the actor for the sake of the story, and also not to believe in the actor for the sake of the actual witness who existed out there but was not the actor. Using the puppet made this contradiction palpable. " ${ }^{46}$

Das Problem testimonialer Überzeugungskraft, wie Kentridge es für das Theater beschreibt, besteht demnach in der doppelten Gefahr, die Zuschauer entweder nicht zu überzeugen - so dass die Darstellung nicht als (künstlerisches) Zeugnis für eine abwesende Wirklichkeit akzeptiert wird - oder sie so sehr zu überzeugen, dass die (notwendig fiktionale) Darstellung die abwesende Wirklichkeit überlagert. Den Einsatz von Puppen begreift Kentridge keineswegs als einfache ,Lösung für

45 Anna Deavere Smith: „The Word Becomes You: An Interview by Carol Martin“, in: TDR: The Drama Review 37 (1993) 4, S. 45-62, hier S. 57.

46 William Kentridge: „Director's Note“, in: Jane Taylor: Ubu and the Truth Commission, Cape Town 2010, S. viii-xv, hier S. xi. 
dieses Problem, sondern vielmehr als Materialisierung des Widerspruchs, der auf eine doppelte Gefährdung des künstlerischen Zeugnisses reagiert. Einerseits betrifft sie dessen potentiell exploitatives Verhältnis zu den eigentlichen Zeugen, andererseits den möglichen Verlust testimonialer Überzeugungskraft im künstlerischen Rahmen.

Kentridge versteht die Puppe als ästhetisches Objekt, das diesbezüglich drei Funktionen erfüllt: Erstens spricht der Regisseur ihr die Kraft zu, ein Zeugnis glaubhaft zu übermitteln. Ihre Überzeugungsmacht liegt dabei darin, dass sie keine volle Autonomie gewinnt. So verweist die Puppe zweitens auf die Differenz zwischen ihr und den eigentlichen Zeugen: „The puppet becomes the medium through which the testimony can be heard. ${ }^{47}$ Drittens ist die Puppe mehr als nur die Instanz, durch die das Zeugnis gesprochen wird, da sie eine konkret in Szene gesetzte Reflexionsebene einführt.

Bei den Aussagen der Opferzeugen verfolgt Ubu and the Truth Commission eine Offenlegung des Spiels: Je zwei sichtbar wahrnehmbare Puppenspieler manipulieren eine der Zeugenpuppen. Diese haben einen starren Gesichtsausdruck, d.h. weder ihre Münder noch ihre Augen lassen sich öffnen und schließen. Wie bei der Truth and Reconciliation Commission (TRC) erfolgt die Aussage zunächst in einer der elf offiziellen Sprachen Südafrikas - in den Opferszenen von Ubu and the Truth Commission ist das jeweils Xhosa - und dann in Übersetzung. Auf diese Weise wird ein dritter menschlicher Akteur in Beziehung zu den Zeugenpuppen gesetzt, der als Übersetzer (ins Englische) fungiert. Die Aussage in Xhosa wird, parallel zu entsprechenden Bewegungen der Gesamtpuppe - aber ohne Veränderung ihres Gesichtsausdrucks - von einem der beiden Puppenspieler gesprochen.

Ubu and the Truth Commission inszeniert hier einen Stimmgebrauch, der die Zeugenaussage in eine Art Schwebezustand zwischen den (auf der Bühne) abwesenden Opferzeugen, den Zeugenpuppen, den sichtbaren Puppenspielern und den Übersetzern bringt. Im Gegensatz zu einem Schauspieler, der die Rolle des Opferzeugen - egal ob distanziert oder illusionistisch - übernehmen würde, hat der Puppenspieler, der die Aussage des Opferzeugen wiederholt, eine sichtbare Verantwortung gegenüber der Puppe als dritter Instanz. Im Figurentheater mit offener Manipulation, schreibt die Theaterwissenschaftlerin und Künstlerin Anna Furse:

„puppeteers are unlike any other actors because they are entirely committed to the Other. [...] What puppeteers contribute to the art of the performer is an almost ethical question. It is a question of attention and the places that the performer draws attention to and from. Rather than draw attention to themselves, actor-puppeteers devote their attention to guiding ours outside, leading our attention away from their own bodies via the body itself." ${ }^{8}$

47 Ebd., S. xi.

48 Furse, zit. nach Penny Francis: Puppetry: A Reader in Theatre Practice, Basingstoke 2012, S. 27. 
Bei Ubu and the Truth Commission lenken die sichtbaren Körper der Puppenspieler die Aufmerksamkeit auf das Wechselspiel zwischen Puppe ,und Manipulator. Die Stimmen, die die Aussagen der Opferzeugen auf der Bühne wiederholen, sind deutlich an die Puppen gebunden, deren Körper sich glaubhaft zum Sprechrhythmus bewegen. Mit ihren starren Mündern verweigern die Puppen aber jede Illusion, autonome Quelle des Sprechens zu sein. Dadurch verweisen sie auf die Puppenspieler zurück. Weil jene aber nicht für eine Figur sprechen, die sie - wie im dramatischen Theatermodell - selbst darstellen, sondern für eine Figur - die Puppe -, die außerhalb ihrer selbst liegt, erfüllt sich das Sprechen weder in ihnen noch in der Puppe. So wird eine Heteronomie des Sprechens erzeugt, die den Bezug zur außertheatralen Referenz der Puppe, den Opferzeugen, stärkt: „The puppet becomes the medium through which the testimony can be heard. " ${ }^{49}$ Diese Möglichkeit liegt in der Objekthaftigkeit der Puppe selbst begründet. „[Since] the puppet does not add its own living body and memory to the [victim's] story", schreibt Stephanie Marlin-Curiel, ,it succeeds in communicating without appropriating. The puppet ,contains' the testimonial repetition by not fully appropriating the testimony from the memory of the ,real' body. " ${ }^{\circ}$

Durch diese Inszenierung der Opferszenen schlägt Ubu and the Truth Commission ein Modell theatraler Zeugenschaft vor, das auf der sichtbar ausgestellten Differenz von Bühnenfigur und Opferzeuge besteht. Der Riss eröffnet einen Raum, bei dem - wie bei Weiss - das Zeugnis vermeintlich selbst zur Sprache kommt. Anders als bei Weiss nimmt die Produktion der Handspring Puppet Company aber nicht für sich in Anspruch, dass das von ihre inszenierte Zeugnis eine größere Klarheit besäße als das Zeugnis vor Gericht bzw. vor dem TRC-Tribunal. Vielmehr verweist die Künstlichkeit der Puppe - wie bei Milo Raus' Kongo Tribunal - auf die ästhetische Differenz zwischen Theater und Tribunal, um von dieser Position aus zu fragen, ob Versöhnung und Gerechtigkeit angesichts der Verbrechen überhaupt möglich ist; eine Frage, die sich - so die Dramatikerin Jane Taylor - außerhalb des ,Spiels‘ verbietet. ${ }^{51}$

49 Kentridge: „Director’s Note“ (Anm. 46), S. xi.

50 Stephanie Marlin-Curiel: „A Little Too Close to the Truth: Anxieties of Testimony and Confession", in: Ubu and the Truth Commission and The Story I am About to Tell", in: South African Theatre Journal 15 (2001) 1, S. 77-106, hier S. 84.

51 Vgl. Jane Taylor: Ubu and the Truth Commission, Cape Town 2010. Im künstlerischen Kontext, so Taylor, „difficult and potentially volatile questions [...] could be addressed without destabilise $[s i c]$ the fragile legal and political process of the TRC itself" (S. iii). 